\title{
CONVERGENCE AND SUMMABILITY PROPERTIES OF SUBSEQUENCES
}

\section{R. CREIGHTON BUCK ${ }^{1}$ AND HARRY POLLARD}

In this paper we shall discuss the relation of the convergence or $(C, 1)$ summability of a sequence to that of its subsequences. Some analogous questions for subseries have been considered [6]. ${ }^{2}$

Let $\left\{s_{n}\right\}$ be an arbitrary sequence. We can obtain a 1-1 map of its infinite subsequences on the interval $0<t \leqq 1$ as follows. Let $t=a_{1} a_{2} a_{3} \cdots$ be the infinite dyadic expansion of a point $t$ of the interval. Corresponding to this point we select the following subsequence: retain $s_{m}$ if the $m$ th place in the expansion, $a_{m}$, is 1 , and drop it otherwise. The inverse correspondence is evident.

In terms of the Lebesgue measure of sets of points on $(0,1)$ we are now clearly in a position to speak of "almost all" or "almost none" of the subsequences of $\left\{s_{n}\right\}$.

The problem we have set ourselves is to determine under what conditions does the convergence or summability of a sequence carry over to that of its subsequences, and conversely, whether these properties for suitable subsequences imply them for the sequence itself. For simplicity, we restrict ourselves to sequences of real numbers; although it is apparent that the results are more generally true.

In the case of convergence, the answer to our problem is simple: a sequence is convergent if and only if almost all of its subsequences are convergent. In the case of $(C, 1)$ summability, the problem is more difficult. It has been established that all the subsequences of a sequence cannot be summable by a fixed regular matrix method unless the sequence is in fact convergent [2]. In $\$ \S 3,4$ we discuss, for $(C, 1)$ summability, the consequences of replacing all by almost all. We show, for example, that $\left\{s_{n}\right\}$ is $(C, 1)$ summable if almost all of its subsequences are, but not conversely.

The principal tools are the Rademacher functions, $R_{n}(t)$, and the properties of homogeneous sets.

Our results have certain obvious connections with probability; these are discussed in $\$ 5$.

1. Preliminary results. All sets which occur in the sequel are to be taken as subsets of $(0,1)$; a.e. will mean "almost everywhere in $(0,1) . "$

Presented to the Society, October 30, 1943; received by the editors May 4, 1943.

1 Society of Fellows, Harvard University.

${ }^{2}$ Numbers in brackets refer to the references listed at the end of the paper. 
We shall call a measurable set $S$ homogeneous if it has the following property: let $t=a_{1} a_{2} a_{3} \cdots$ be the infinite dyadic expansion of a point $t$ of $S$; then the point obtained by altering a finite number of the $a_{i}$ also belongs to $S$. This definition is more restricted than the usual one [7], but is adequate for our purposes.

\section{Lemma 1. A homogeneous set has measure 0 or 1.}

This is a well known result $[7$, p. 145]. The sets with which our theorems deal are homogeneous. In view of this lemma, the frequently occurring phrase "almost all the subsequences" can be replaced by the seemingly weaker but actually equivalent phrase "the subsequences corresponding to a set of positive measure."

LEMмA 2. If $S$ is of measure 1, then there exists a subset E, also of measure 1 , with the property that if $t$ belongs to $E$, so does $1-t$.

This becomes immediately evident if the interval $(0,1 / 2)$ is folded symmetrically over onto the interval $(1 / 2,1)$. The device of symmetric points was used to advantage in [6].

LEMMA 3. Let $p_{n}$ be a sequence of positive numbers, increasing monotonically to infinity. If the series $\sum_{n=1}^{\infty} a_{n} / p_{n}$ converges, then $[5, \mathrm{p} .123]$

$$
\lim _{n \rightarrow \infty} \frac{1}{p_{n}} \sum_{1}^{n} a_{k}=0 .
$$

For the properties of the Rademacher functions, we refer the reader to [3]. The principal one is contained in the following lemma.

LEMмA 4. The series $\sum_{1}^{\infty} a_{k} R_{k}(t)$ converges on a set of measure 1 or measure 0 , according as $\sum_{1}^{\infty} a_{k}^{2}$ converges or diverges.

LEMMA 5. If $\sum_{1}^{\infty} s_{k}^{2} / k^{2}$ converges, then

$$
\lim _{n \rightarrow \infty} \frac{1}{n} \sum_{1}^{n} s_{k} R_{k}(t)=0 \text { a.e. }
$$

For the hypothesis implies, by Lemma 4 , that $\sum_{1}^{\infty}\left(s_{k}^{2} / k^{2}\right) R_{k}(t)$ converges a.e., so that the result follows from Lemma 3 , with $p_{n}=n$.

LEMMA 6. If

$$
\lim _{n \rightarrow \infty} \frac{1}{n} \sum_{1}^{n} s_{k} R_{k}(t)=L(t)
$$

exists a.e., then $L(t)=0$ a.e. (so that (1.1) is true) and 


$$
\lim _{n \rightarrow \infty} \frac{1}{n^{2}} \sum_{1}^{n} s_{k}^{2}=0 .
$$

$\left(1.1^{\prime}\right)$ must hold uniformly on a set $A$ of positive measure. Hence, for some $M$,

$$
|L(t)| \leqq M, \quad t \in A .
$$

But the set of points for which this inequality holds is homogeneous, so that, by Lemma 1, $L(t)$ is bounded a.e. Since it is obviously integrable,

$$
\int_{0}^{1} L(t) d t=a
$$

exists.

Now, choose an $\epsilon>0$ and consider the sets $I_{1}, I_{2}$ and $I_{3}$ for which

$$
\begin{array}{r}
L(t)-a>\epsilon, \\
L(t)-a<-\epsilon, \\
|L(t)-a| \leqq \epsilon,
\end{array}
$$

respectively. These sets are homogeneous; since their union is of measure 1, at least one of them must be of measure 1. If either $I_{1}$ or $I_{2}$ has measure $1,(1.3)$ is contradicted. Hence $|L(t)-a| \leqq \epsilon$ a.e., so that $L(t)=a$ on a set $B$ of measure 1 .

By Lemma 2, we can pick two points $t_{0}$ and $1-t_{0}$ in $B$. By $\left(1.1^{\prime}\right)$

$$
\begin{aligned}
& \lim _{n \rightarrow \infty} \frac{1}{n} \sum_{1}^{n} s_{k} R_{k}\left(t_{0}\right)=a, \\
& \lim _{n \rightarrow \infty} \frac{1}{n} \sum_{1}^{n} s_{k} R_{k}\left(1-t_{0}\right)=a .
\end{aligned}
$$

Adding these, we see that $a=0$; this establishes (1.1).

To prove (1.2) we employ a device due to Kolmogoroff [4, p. 127]. Let $S_{m, n}(t)=\sum_{m}^{n} s_{k} R_{k}(t)$. Then

$$
\begin{aligned}
S_{m, n}^{2}(t) & =\sum_{j, k=m}^{n} s_{j} s_{k} R_{j}(t) R_{k}(t) \\
& =\sum_{m}^{n} s_{k}^{2}+2 \sum_{m \leqq j<k \leqq n} s_{j} s_{k} R_{j}(t) R_{k}(t) .
\end{aligned}
$$

If (1.1) holds for almost all $t$, it must hold uniformly on a set $E$ of positive measure $|E|$. By (1.4) 


$$
\int_{E} S_{m, n}^{2}(t) d t=|E| \sum_{m}^{n} s_{k}^{2}+M
$$

where

$$
M=2 \sum_{m \leqq j<k \leqq n} s_{j} s_{k} \int_{E} R_{j}(t) R_{k}(t) d t .
$$

From the Schwarz inequality

$$
|M| \leqq 2\left(\sum_{m \leqq j<k \leqq n} s_{j}^{2} s_{k}^{2}\right)^{1 / 2}\left(\sum_{m \leqq j<k \leqq n} b_{j k}^{2}\right)^{1 / 2}
$$

where $b_{j k}=\int_{E} R_{j}(t) R_{k}(t) d t$.

The functions $R_{j}(t) R_{k}(t)$, for $1 \leqq j<k<\infty$, are orthonormal on $(0,1)$. If $X(t)$ is the characteristic function of $E$, it follows from Bessel's inequality that

$$
\sum_{1 \leqq j<k<\infty} b_{j k}^{2} \leqq \int_{0}^{1}[X(t)]^{2} d t=|E| .
$$

For a large enough value of $m$

$$
\left(\sum_{m \leqq j<k<\infty} b_{j k}^{2}\right)^{1 / 2} \leqq|E| / 4 .
$$

By (1.6)

$$
|M| \leqq\left(\sum_{j, k=m}^{n} s_{j}^{2} s_{k}^{2}\right)^{1 / 2}(|E| / 2) \leqq(|E| / 2) \sum_{m}^{n} s_{j}^{2} .
$$

Then, by (1.5) we have

$$
\int_{E} S_{m, n}^{2}(t) d t \geqq(|E| / 2) \sum_{m}^{n} s_{j}^{2}
$$

But for this fixed value of $m$,

$$
\lim _{n \rightarrow \infty} \frac{1}{n^{2}} S_{m, n}^{2}(t)=0
$$

boundedly on $E$. (1.7) then implies that

$$
\lim _{n \rightarrow \infty} \frac{1}{n^{2}} \sum_{m}^{n} s_{j}^{2}=0
$$

and the proof is complete. 
2. Convergence of subsequences. If a sequence $\left\{s_{n}\right\}$ of real numbers is convergent to $S$, then every subsequence is likewise convergent to $S$. What may be said if $\left\{s_{n}\right\}$ is divergent?

THEOREM 1. If $\left\{s_{n}\right\}$ is divergent, so are almost all of its subsequences.

Let $C$ be the set of points corresponding to convergent subsequences. It is homogeneous, and hence of measure 0 or 1 . Suppose it is of measure 1. By Lemma 2, we can pick a number $t_{0}$ not of the form $K / 2^{n}$, such that both $t_{0}$ and $1-t_{0}$ belong to $C$. The corresponding subsequences can have no terms in common, for the dyadic expansions of $t_{0}$ and $1-t_{0}$ cannot agree anywhere. Furthermore, every term of $\left\{s_{n}\right\}$ is present in one or the other of the subsequences. Thus we have split $\left\{s_{n}\right\}$ in to two subsequences, and since $t_{0} \in C, 1-t_{0} \in C$, each is convergent. Let their limit points be $S^{\prime}$ and $S^{\prime \prime}$.

Every convergent subsequence of $\left\{s_{n}\right\}$ must then have either $S^{\prime}$ or $S^{\prime \prime}$ as its limit. Let $C^{\prime}\left(C^{\prime \prime}\right)$ be the set of points corresponding to subsequences convergent to $S^{\prime}\left(S^{\prime \prime}\right)$. Then $C^{\prime}$ and $C^{\prime \prime}$ are homogeneous and, since $C=C^{\prime} \cup C^{\prime \prime}$, one of them-say $C^{\prime}$ - must be of measure 1. This means that the $t_{0}$ above could have been chosen from $C^{\prime}$, so that $\left\{s_{n}\right\}$ can be split into two convergent subsequences, each of which converges to the same limit. Every subsequence is then convergent, and hence $\left\{s_{n}\right\}$ is itself convergent; this contradicts the hypothesis of our theorem, and $C$ therefore has measure 0 .

COROLLARY. If the series $\sum_{1}^{\infty} a_{k}$ converges for almost every bracketing of terms, it is convergent.

For if $\left\{s_{n}\right\}$ is the sequence of partial sums, and we bracket the series in blocks of length $n_{1}, n_{2}, \cdots$, then the partial sums of the resulting series are $s_{n_{1}}, s_{n_{1}+n_{2}}, \cdots$, a subsequence of $\left\{s_{n}\right\}$. By the theorem, if almost all of these converge, the sequence itself does.

3. $(C, 1)$ summability. In this and the succeeding section, we consider the corresponding problem for $(C, 1)$ summability. As we have previously noted, we cannot expect all of the subsequences of a sequence to be $(C, 1)$ summable unless the sequence is convergent.

THEOREM 2. If $\left\{s_{n}\right\}$ is $(C, 1)$ summable to $S$, and

$$
\sum_{1}^{\infty} s_{k}^{2} / k^{2}<\infty
$$

then almost all the subsequences are $(C, 1)$ summable to $S$. 
The problem of the convergence of the Cesàro means of almost all the subsequences of $\left\{s_{n}\right\}$ reduces to the requirement that

$$
\lim _{n \rightarrow \infty} \frac{\sum_{1}^{n} s_{k} \cdot \frac{1}{2}\left[1+R_{k}(t)\right]}{\sum_{1}^{n} \frac{1}{2}\left[1+R_{k}(t)\right]}=S \text { a.e. }
$$

We can rewrite the expression inside the limit as

$$
\frac{\frac{1}{n} \sum_{1}^{n} s_{k}+\frac{1}{n} \sum_{1}^{n} s_{k} R_{k}(t)}{1+\frac{1}{n} \sum_{1}^{n} R_{k}(t)} .
$$

By hypothesis, $\lim (1 / n) \sum_{1}^{n} s_{k}=S$; then (3.2) follows immediately by condition (3.1) and Lemma 4.

The question arises whether the condition (3.1) can be removed or at least weakened. We shall show in the next section that it cannot be dropped entirely; on the other hand, this does not preclude the possibility that it might be weakened, perhaps to (1.2) which by Lemma 3 it implies, and which is necessary for the validity of the conclusion (Theorem 3 , corollary).

4. Converse. We now prove the analogue of Theorem 1 for $(C, 1)$ summability. We note again that since $(C, 1)$ summability is a method that preserves translation, our sets are homogeneous.

Theorem 3. If almost all the subsequences of $\left\{s_{n}\right\}$ are $(C, 1)$ summable, then $\left\{s_{n}\right\}$ is itself summable to a value $S$, and almost all the subsequences are in turn summable to $S$.

As in the preceding section, the hypothesis implies that (3.3) converges a.e. as $n \rightarrow \infty$. By Lemma 5, the denominator of (3.3) approaches the value 1 a.e., so that

$$
\sigma_{n}(t)=\frac{1}{n} \sum_{1}^{n} s_{k}\left[1+R_{k}(t)\right]
$$

converges on a set $C$ of measure 1. By Lemma 2, we can pick $t_{0}$ and $1-t_{0}$ belonging to $C$; then

$$
\sigma_{n}\left(t_{0}\right)+\sigma_{n}\left(1-t_{0}\right)=\frac{2}{n} \sum_{1}^{n} s_{k}
$$


so that $(1 / n) \sum_{1}^{n} s_{k}$ converges to a value $S$. From (4.1) we see that

$$
\frac{1}{n} \sum_{1}^{n} s_{k} R_{k}(t)
$$

converges a.e.; by Lemma 6, this last expression then converges a.e. to 0 , and, by (4.1),

$$
\lim \sigma_{n}(t)=S \text { a.e. }
$$

COROLLARY. Under the hypothesis of the theorem,

$$
\lim _{n \rightarrow \infty} \frac{1}{n^{2}} \sum_{1}^{n} s_{k}^{2}=0 .
$$

For (4.2) converges a.e.; (4.3) follows from Lemma 6.

We are now in a position to answer the question raised in the preceding section by exhibiting a $(C, 1)$ summable sequence with almost none of its subsequences $(C, 1)$ summable. This example is the sequence $s_{n}=(-1)^{n} n^{1 / 2}$. It is readily seen to be summable to zero, but since it violates $(4.3)$, almost none of its subsequences can be $(C, 1)$ summable.

If the sequence $\left\{s_{n}\right\}$ is bounded, condition (3.1) is satisfied. Combining Theorems 2 and 3, we have the following theorem.

THEOREM 4. $A$ bounded sequence is $(C, 1)$ summable if and only if almost all of its subsequences are $(C, 1)$ summable.

5. Probability. Lemma 5, on which a large part of our paper depends, can also be obtained from the strong law of large numbers $\left[4\right.$, p. 59], for we may regard the sequence $s_{n} R_{n}(t)$ as a sequence of independent random variables of mean value 0 and standard deviation $s_{n^{2}}$.

An interesting result that may be obtained as a corollary of Theorem 2 is one of Birnbaum and Zuckerman on the v. Mises collective [1].

THEOREM A. If a sequence of 0's and 1's satisfies the first postulate of $v$. Mises, the second postulate is fulfilled for almost all selections.

Here the v. Mises postulates are:

$\mathrm{P} 1$ : If $n_{0}$ and $n_{1}$ are the number of 0 's and 1's respectively among the first $n$ terms of the sequence, then the limits $\lim n_{0} / n$ and $\lim n_{1} / n$ exist.

P2: For a suitable selection of an infinite subsequence from the given sequence, the same limits exist and their values are unchanged.

To obtain the theorem we need only observe that $\lim n_{1} / n$ is the 
$(C, 1)$ sum of the given sequence, and that (3.1) is automatically satisfied.

\section{REFERENCES}

1. Birnbaum and Zuckerman, On the properties of a collective, Amer. J. Math. vol. 62 (1940) pp. 787-791.

2. R. Creighton Buck, $A$ note on subsequences, Bull. Amer. Math. Soc. vol. 49 (1943) pp. 898-899.

3. Kacmarz and Steinhaus, Theorie der orthogonalreihen, Warsaw, 1935, pp. 125132.

4. A. Kolmogoroff, Grundbegriffe der Wahrscheinlichskeitsrechnung, Ergebnisse der Mathematik und ihrer grenzgebiete, vol. 2, no. 3, Berlin, 1933.

5. K. Knopp, Theorie und Anwendung der unendlichen Reihen, Berlin, 1922.

6. H. Pollard, Subseries of a convergent series, Bull. Amer. Math. Soc. vol. 49 (1943) pp. 730-731.

7. C. Visser, The law of nought-or-one, Studia Mathematica vol. 7 (1938) pp. 143-159.

HARVARD UNIVERSITY 